\title{
Distributed Information Consensus Filters for Simultaneous Input and State Estimation
}

\author{
Ying Lu · Liguo Zhang · Xuerong Mao
}

Received: date / Accepted: date

\begin{abstract}
This paper describes the distributed information filtering where a set of sensor networks are required to simultaneously estimate input and state of a linear discrete-time system from collaborative manner. Our research purpose is to develop a consensus strategy in which sensor nodes communicate within the network through a sequence of Kalman iterations and data diffusion. A novel recursive information filtering is proposed by integrating input estimation error into measurement data and weighted information matrices. On the fusing process, local system state filtering transmits estimation information using the consensus averaging algorithm, which penalizes the disagreement in a dynamic manner. A simulation example is provided to compare the performance of the distributed information filtering with optimal Gillijins-De Moor's algorithm.
\end{abstract}

Keywords Unknown input estimation · Distributed estimation · Information filters · Consensus · Sensor networks

Y. Lu

Department of Sciences, Communication University of China, Beijing, 100024, PR China School of Applied Science, Beijing University of Technology, Beijing, 100124, PR China E-mail: ly@cuc.edu.cn

L. Zhang, Corresponding Author

School of Electronic Information and Control Engineering, Beijing University of Technology, Beijing, 100124, PR China

E-mail: zhangliguo@bjut.edu.cn

Present address: System Engineering Group, Department of Civil and Environmental Engineering, University of California, Berkeley, CA 94720, USA

E-mail: zhangliguo@berkeley.edu

X. Mao

Department of Mathematics and Statistics, University of Strathclyde, Glasgow, G1 1XH, UK

E-mail: x.mao@strath.ac.uk 


\section{Introduction}

Simultaneous input and state estimation for dynamic systems has a wide range of applications, ranging from fault detection and isolation [13], geophysical and environment surveillance [22], maneuvering target tracking [11], to video watermarking protection [15-18], etc. In these applications, input variables are often unmeasurable and inaccessible and treated as a stochastic process with unknown statistics. This problem can also find applications in estimation of the large-scale distributed parameter systems with a more general consideration that no prior information about boundary conditions are available [28]. Because of its practical significance, simultaneous input and state estimation has received considerable attention during the past several decades.

We consider the distributed filtering for simultaneous input and state estimation over a sensor network, in which each sensor node might access to the different local measurement depending on the available resources. Basic principles and structures for distributed estimation have been discussed in [25], [26]. One of the general design methodologies have been derived from the consensus protocols [4], [9], [27]. The considered consensus filtering involves design of information processing algorithm with a two-fold objective. One is every node jointly estimate the input and the state variables of systems based on local observations, the other is update the local filtering information to reach a consensus by including the estimation received from its neighboring sensor nodes. The research of consensus protocols for spatially fixed or mobile sensor networks has been considered extensively in the context of distributed state estimation, coverage control and target tracking [19-21], etc.

On the other hand, optimal filtering for joint input and state estimation is a challenging task since they are inherently interconnected and coupled. An optimal recursive state filter is developed in [20] without direct feedthrough of the unknown input to the measurement. In [8], a two-stage Kalman filter and an optimal input filtering technique are combined to achieve joint input and state estimation. Most recently, in [6], [7], a set of muliti-step recursive filters are proposed by minimizing the error variance in the absence and presence the direct feedthrough, respectively. In addition minimum variance estimation, some matrix manipulation methods are useful for state estimation with unknown inputs, for instance, in [5], [23] and reference therein. In spite of many optimal filtering algorithms have been extended, to the best of the authors knowledge, information filtering approach has not been developed for simultaneous input and state estimation.

In this paper, a novel distributed information consensus filtering for for simultaneous input and state estimation of linear discrete-time systems is proposed based on:

(1) to develop a recursive information filtering for simultaneous input and state estimation by combining the input estimation error into the weighted measurement data and the weighted information matrix. This kind of filter is characterized by computation of the inverse of the covariance matrix and the state information estimate. 
(2) to design a consensus protocol in which the local partially state estimates transmit their information using consensus averaging algorithm and penalize the disagreement among themselves in a dynamic manner. The proposed algorithm presents an extension of the distributed Kalman consensus filter proposed in [21] by joint input and state estimation.

The subsequent sections are organized as follows. Some background on sensor networks and simultaneous input and state estimation method are provided in Section 2. Section 3 studies the extended information filtering algorithm with unknown input. Distributed information consensus filtering over sensor networks is introduced in Section 4, and a numerical example for performance comparison is presented in Section 5.

\section{Problem formulation}

Consider the following linear discrete-time stochastic time-varying model with unknown inputs:

$$
x_{k+1}=A_{k} x_{k}+B_{k} d_{k}+w_{k}
$$

where, $x_{k} \in R^{n}, d_{k} \in R^{m}$, and $w_{k} \in R^{n}$ are the state vector, unknown input, and process noise at time $k \in\{0,1,2, \cdots\}$, respectively. $A_{k}, B_{k}$ are deterministic known matrices with appropriate dimensions. $w_{k}$ are uncorrelated white noise with covariance matrices $Q_{k}=E\left[w_{k} w_{k}^{T}\right]>0$.

In the distributed approach, we are interested in tracking the input and state of this process using a network of $N$ sensors. Each sensor $i$ measures the partial observations as

$$
z_{k}^{(i)}=H_{k}^{(i)} x_{k}+v_{k}^{(i)}
$$

where $z_{k}^{(i)} \in R^{p_{i}}$, with $\sum_{i=1}^{N} p_{i}=p$, and $v_{k}^{(i)}$ is measurement noise assumed to be white noise with known covariance matrices $R_{k}^{(i)}=E\left[v_{k}^{(i)} v_{k}^{(i) T}\right]>0$.

We collect overall observations in the sensor network to get a centralized observation model. Let observation vector $z_{k} \in R^{p}$, observation matrix $H_{k} \in$ $R^{p \times n}$, and observation noise vector $v_{k} \in R^{p}$ be

$$
\begin{aligned}
& z_{k}=\left[z_{k}^{(1)}, \cdots, z_{k}^{(N)}\right]^{T}, \\
& H_{k}=\left[H_{k}^{(1)}, \cdots, H_{k}^{(N)}\right]^{T} \text {, } \\
& v_{k}=\left[v_{k}^{(1)}, \cdots, v_{k}^{(N)}\right]^{T} .
\end{aligned}
$$

Then the centralized observation model is given by

$$
z_{k}=H_{k} x_{k}+v_{k}
$$

Since observation noises of different sensors are mutually independent, we can combine $R_{k}^{(i)}$ into one global covariance matrix $R_{k}$, as

$$
R_{k}=\operatorname{blockdiag}\left[R_{k}^{(1)}, \cdots, R_{k}^{(N)}\right]
$$


Satisfied the assumption [6] that $\operatorname{rank} H_{k} B_{k-1}=\operatorname{rank} B_{k-1}=m$, for all $k$, the recursive joint input and state filters for system $(1),(4)$ are as follows:

$$
\begin{aligned}
\hat{x}_{k \mid k} & =\bar{x}_{k \mid k}+K_{k}\left(z_{k}-H_{k} \bar{x}_{k \mid k}\right), \\
\bar{x}_{k \mid k} & =\hat{x}_{k \mid k-1}+B_{k-1} \hat{d}_{k-1}, \\
\hat{x}_{k \mid k-1} & =A_{k-1} \hat{x}_{k-1 \mid k-1}, \\
\hat{d}_{k-1} & =M_{k}\left(z_{k}-H_{k} \hat{x}_{k \mid k-1}\right),
\end{aligned}
$$

where $\hat{x}_{k \mid k}$ and $\hat{d}_{k-1}$ represent the state and input estimate, $\bar{x}_{k \mid k}$ and $\hat{x}_{k \mid k-1}$ represent the state prediction with or without the input information. $K_{k} \in$ $R^{n \times p}, M_{k} \in R^{p \times m}$ are the gain matrices to be determined.

The error covariance matrices of system (1) are defined as

$$
\begin{gathered}
P_{k \mid k}^{+}=E\left[\eta_{k} \eta_{k}^{T}\right], \\
P_{k \mid k}^{-}=E\left[\bar{\eta}_{k} \bar{\eta}_{k}^{T}\right],
\end{gathered}
$$

where $\eta_{k}=x_{k}-\hat{x}_{k \mid k}, \bar{\eta}_{k}=x_{k}-\bar{x}_{k \mid k}$. It is straightforward to see that matrices $P_{k \mid k}^{+}, P_{k \mid k}^{-}$are symmetric positive definite.

By minimizing the error variance, the optimal gain matrices are obtained as

$$
\begin{aligned}
M_{k} & =\left(D_{k}^{T} \tilde{R}_{k}^{-1} D_{k}\right)^{-1} D_{k}^{T} \tilde{R}_{k}^{-1}, \\
K_{k} & =\left(P_{k \mid k}^{-} H_{k}^{T}+S_{k}\right) \breve{R}_{k}^{-1},
\end{aligned}
$$

where $D_{k}=H_{k} B_{k-1}, S_{k}=-B_{k-1} M_{k} R_{k}$,

$$
\tilde{R}_{k}=H_{k}\left(A_{k-1} P_{k-1 \mid k-1}^{+} A_{k-1}^{T}+Q_{k}\right) H_{k}^{T}+R_{k}
$$

and

$$
\breve{R}_{k}=H_{k} P_{k \mid k}^{-} H_{k}^{T}+R_{k}+H_{k} S_{k}+S_{k}^{T} H_{k}^{T} .
$$

The error covariance matrices update according to

$$
\begin{aligned}
& P_{k \mid k}^{+}=P_{k \mid k}^{-}-K_{k}\left(P_{k \mid k}^{-} H_{k}^{T}+S_{k}\right)^{T}, \\
& P_{k \mid k}^{-}=\tilde{A}_{k-1} P_{k-1 \mid k-1}^{+} \tilde{A}_{k-1}^{T}+\tilde{Q}_{k-1},
\end{aligned}
$$

where,

$$
\begin{aligned}
& \tilde{A}_{k-1}=\left(I_{n}-B_{k-1} M_{k} H_{k}\right) A_{k-1}, \\
& \tilde{Q}_{k-1}=B_{k-1} M_{k} R_{k} M_{k}^{T} B_{k-1}^{T} .
\end{aligned}
$$

Remark 1: For any $k, \breve{R}_{k}$ is singular in Gillijins-De Moor's algorithm which means that the optimal gain $K_{k}$ is not unique. However, here it could be assumed nonsingular only by choosing its full rank submatrix and maintains the above equations. 


\section{Information filters for simultaneous input and state estimation}

In this section, we extend Kalman filtering (6)-(9) to information filtering by propagating the inverse of the estimation error covariance matrix rather than the error covariance itself.

Recall from equation (11) that the Kalman gain $K_{k}$ could be written as

$$
\begin{aligned}
K_{k}= & \left(P_{k \mid k}^{-} H_{k}^{T}+S_{k}\right) \breve{R}_{k}^{-1} \\
= & P_{k \mid k}^{-}\left[H_{k}+S_{k}^{T}\left(P_{k \mid k}^{-}\right)^{-1}\right]^{T} \breve{R}_{k}^{-1} \\
= & P_{k \mid k}^{-}\left[H_{k}+S_{k}^{T}\left(P_{k \mid k}^{-}\right)^{-1}\right]^{T}\left[H_{k} P_{k \mid k}^{-} H_{k}^{T}+R_{k}+H_{k} S_{k}+S_{k}^{T} H_{k}^{T}\right]^{-1} \\
= & P_{k \mid k}^{-}\left[H_{k}+S_{k}^{T}\left(P_{k \mid k}^{-}\right)^{-1}\right]^{T}\left[\left(H_{k}+S_{k}^{T}\left(P_{k \mid k}^{-}\right)^{-1}\right) P_{k \mid k}^{-}\right. \\
& \left.\left(H_{k}+S_{k}^{T}\left(P_{k \mid k}^{-}\right)^{-1}\right)^{T}+R_{k}-S_{k}^{T}\left(P_{k \mid k}^{-}\right)^{-1} S_{k}\right]^{-1} .
\end{aligned}
$$

Defining

$$
\begin{gathered}
\bar{H}_{k}=H_{k}+S_{k}^{T}\left(P_{k \mid k}^{-}\right)^{-1}, \\
\bar{R}_{k}=R_{k}-S_{k}^{T}\left(P_{k \mid k}^{-}\right)^{-1} S_{k},
\end{gathered}
$$

then the Kalman gain matrix can be equivalently expressed as

$$
K_{k}=P_{k \mid k}^{-} \bar{H}_{k}^{T}\left(\bar{H}_{k} P_{k \mid k}^{-} \bar{H}_{k}^{T}+\bar{R}_{k}\right)^{-1}
$$

and the error covariance matrix $P_{k \mid k}^{+}$can be expressed as

$$
\begin{aligned}
P_{k \mid k}^{+} & =P_{k \mid k}^{-}-K_{k}\left(P_{k \mid k}^{-} H_{k}^{T}+S_{k}\right)^{T} \\
& =P_{k \mid k}^{-}-K_{k}\left[H_{k}+S_{k}^{T}\left(P_{k \mid k}^{-}\right)^{-1}\right] P_{k \mid k}^{-} \\
& =P_{k \mid k}^{-}-K_{k} \bar{H}_{k} P_{k \mid k}^{-} \\
& =P_{k \mid k}^{-}-P_{k \mid k}^{-} \bar{H}_{k}^{T}\left(\bar{H}_{k} P_{k \mid k}^{-} \bar{H}_{k}^{T}+\bar{R}_{k}\right)^{-1} \bar{H}_{k} P_{k \mid k}^{-} .
\end{aligned}
$$

Taking the inverse of both sides of above equation gives

$$
\left(P_{k \mid k}^{+}\right)^{-1}=\left[P_{k \mid k}^{-}-P_{k \mid k}^{-} \bar{H}_{k}^{T}\left(\bar{H}_{k} P_{k \mid k}^{-} \bar{H}_{k}^{T}+\bar{R}_{k}\right)^{-1} \bar{H}_{k} P_{k \mid k}^{-}\right]^{-1} .
$$

Applying the matrix inversion lemma,

$$
\left(A+B D^{-1} C\right)^{-1}=A^{-1}-A^{-1} B\left(D+C A^{-1} B\right)^{-1} C A^{-1},
$$

equation (19) can be written as

$$
P_{k \mid k}^{+}=\left[\left(P_{k \mid k}^{-}\right)^{-1}+\bar{H}_{k}^{T} \bar{R}_{k}^{-1} \bar{H}_{k}\right]^{-1} .
$$


Substituting $\left(P_{k \mid k}^{+}\right)^{-1}$ into equation (18), we have

$$
\begin{aligned}
K_{k} & =P_{k \mid k}^{+}\left[\left(P_{k \mid k}^{-}\right)^{-1}+\bar{H}_{k}^{T} \bar{R}_{k}^{-1} \bar{H}_{k}\right] P_{k \mid k}^{-} \bar{H}_{k}^{T}\left(\bar{H}_{k} P_{k \mid k}^{-} \bar{H}_{k}^{T}+\bar{R}_{k}\right)^{-1} \\
& =P_{k \mid k}^{+}\left(\bar{H}_{k}^{T}+\bar{H}_{k}^{T} \bar{R}_{k}^{-1} \bar{H}_{k} P_{k \mid k}^{-} \bar{H}_{k}^{T}\right)\left(\bar{H}_{k} P_{k \mid k}^{-} \bar{H}_{k}^{T}+\bar{R}_{k}\right)^{-1} \\
& =P_{k \mid k}^{+} \bar{H}_{k}^{T}\left(I+\bar{R}_{k}^{-1} \bar{H}_{k} P_{k \mid k}^{-} \bar{H}_{k}^{T}\right)\left(\bar{H}_{k} P_{k \mid k}^{-} \bar{H}_{k}^{T}+\bar{R}_{k}\right)^{-1} \\
& =P_{k \mid k}^{+} \bar{H}_{k}^{T} \bar{R}_{k}^{-1}\left(\bar{R}_{k}+\bar{H}_{k} P_{k \mid k}^{-} \bar{H}_{k}^{T}\right)\left(\bar{H}_{k} P_{k \mid k}^{-} \bar{H}_{k}^{T}+\bar{R}_{k}\right)^{-1} \\
& =P_{k \mid k}^{+} \bar{H}_{k}^{T} \bar{R}_{k}^{-1}
\end{aligned}
$$

which gives the Kalman gain for joint input and state estimation with correlated error covariance.

Similar to the derivation of formula (21), we further have

$$
P_{k \mid k}^{+}=\left(I-K_{k} \bar{H}_{k}\right) P_{k \mid k}^{-}\left(I-K_{k} \bar{H}_{k}^{T}\right)+K_{k} \bar{R}_{k} K_{k}^{T},
$$

which gives measurement update equation for estimation error covariance with correlated process and measurement noise.

Substituting $\bar{H}_{k}$ into equation (6) gives

$$
\begin{aligned}
\hat{x}_{k \mid k} & =\bar{x}_{k \mid k}+K_{k}\left(z_{k}-H_{k} \bar{x}_{k \mid k}\right) \\
& =\bar{x}_{k \mid k}+K_{k}\left[z_{k}+S_{k}^{T}\left(P_{k \mid k}^{-}\right)^{-1} \bar{x}_{k \mid k}-\bar{H}_{k} \bar{x}_{k \mid k}\right] .
\end{aligned}
$$

Define the weighted sensor measurement $y_{k}$ and the information matrix $U_{k}$ as

$$
\begin{aligned}
y_{k} & =\bar{H}_{k}^{T} \bar{R}_{k}^{-1}\left[z_{k}+S_{k}^{T}\left(P_{k \mid k}^{-}\right)^{-1} \bar{x}_{k \mid k}\right], \\
U_{k} & =\bar{H}_{k}^{T} \bar{R}_{k}^{-1} \bar{H}_{k},
\end{aligned}
$$

then, the state estimation equation and error covariance matrix can be restated as

$$
\begin{aligned}
& \hat{x}_{k \mid k}=\bar{x}_{k \mid k}+P_{k \mid k}^{+}\left(y_{k}-U_{k} \bar{x}_{k \mid k}\right), \\
& P_{k \mid k}^{+}=\left[\left(P_{k \mid k}^{-}\right)^{-1}+U_{k}\right]^{-1} .
\end{aligned}
$$

Information filtering for simultaneous input and state estimation can be summarized as follows :

$$
\begin{aligned}
\hat{x}_{k \mid k} & =\bar{x}_{k \mid k}+P_{k \mid k}^{+}\left(y_{k}-U_{k} \bar{x}_{k \mid k}\right), \\
U_{k} & =\bar{H}_{k}^{T} \bar{R}_{k}^{-1} \bar{H}_{k}, \\
P_{k \mid k}^{+} & =\left[\left(P_{k \mid k}^{-}\right)^{-1}+U_{k}\right]^{-1}, \\
P_{k \mid k}^{-} & =\tilde{A}_{k-1} P_{k-1 \mid k-1}^{+} \tilde{A}_{k-1}^{T}+\tilde{Q}_{k-1}, \\
\bar{x}_{k \mid k} & =\hat{x}_{k \mid k-1}+B_{k-1} \hat{d}_{k-1}, \\
\hat{x}_{k \mid k-1} & =A_{k-1} \hat{x}_{k-1 \mid k-1}, \\
\hat{d}_{k-1} & =M_{k}\left(z_{k}-H_{k} \hat{x}_{k \mid k-1}\right),
\end{aligned}
$$




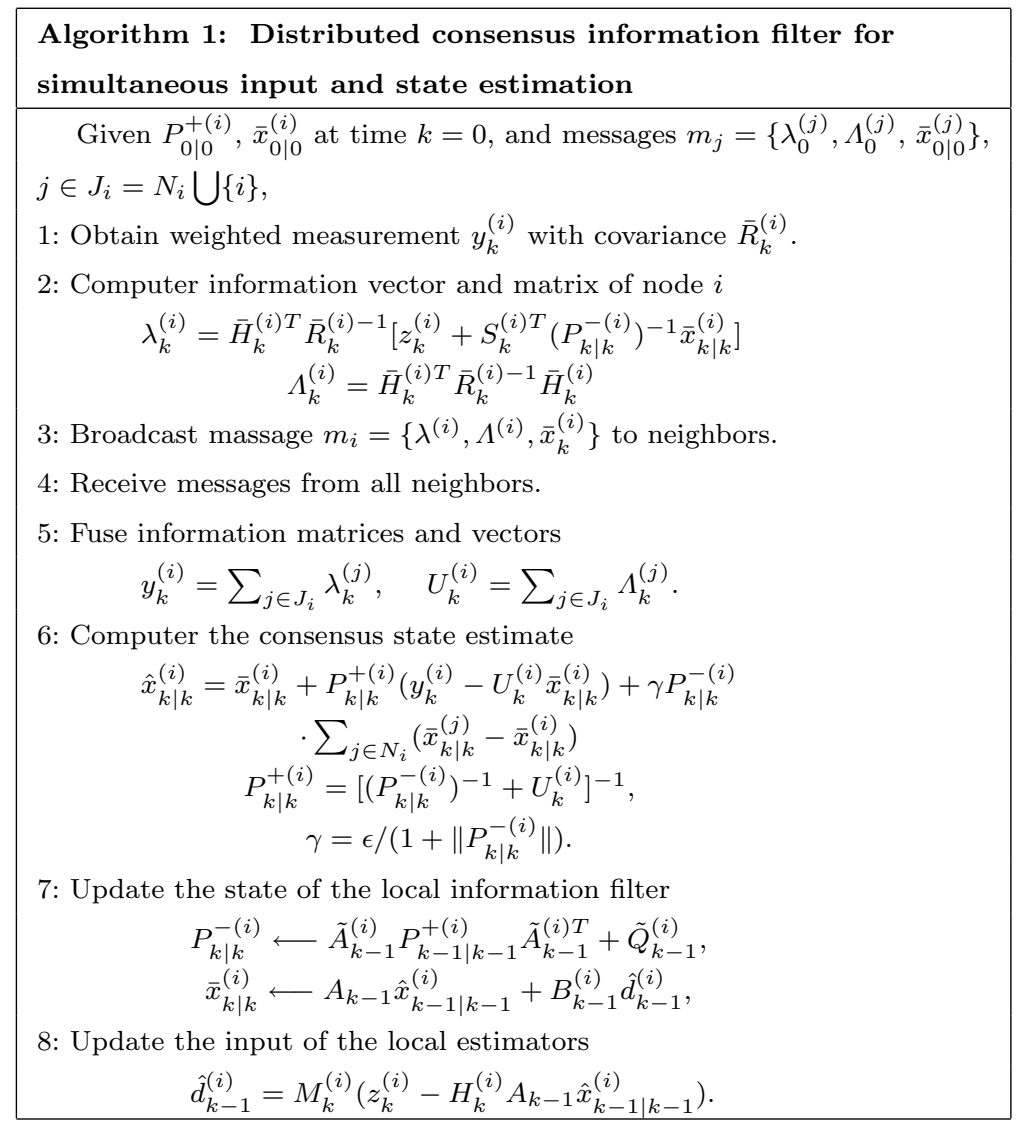

with weighted sensor measurement data $y_{k}$.

Remark 2: For the simultaneous state and input estimation problem [6], if the prior input estimate is unbiased, then in the state estimation step, the Kalman state gain matrix $K_{k}$ and the error covariance matrix $P_{k \mid k}^{+}$have the familiar updating forms of the standard Kalman information filter.

Remark 3: When $B_{k}=0$, the classic information filter without unknown input in the system model is obtained.

\section{Distributed information consensus filter}

We propose a distributed Kalman consensus filtering consisting of local filters with a disagreement term

$$
\hat{x}_{k \mid k}^{(i)}=\bar{x}_{k \mid k}^{(i)}+K_{k}^{(i)}\left(z_{k}^{(i)}-H_{k}^{(i)} \bar{x}_{k \mid k}^{(i)}\right)+C_{k}^{(i)} \sum_{j \in N_{i}}\left(\bar{x}_{k \mid k}^{(j)}-\bar{x}_{k \mid k}^{(i)}\right)
$$

where $K_{k}^{(i)}, C_{k}^{(i)}$ are Kalman and consensus gains of node $i, N_{i}$ denote the neighbors of sensor node $i$. 
We can choose

$$
\begin{aligned}
& K_{k}^{(i)}=P_{k \mid k}^{-(i)} \bar{H}_{k}^{(i) T}\left(\bar{H}_{k}^{(i)} P_{k \mid k}^{-(i)} \bar{H}_{k}^{(i) T}+\bar{R}_{k}^{(i)}\right)^{-1}, \\
& C_{k}^{(i)}=\gamma P_{k \mid k}^{-(i)}=\epsilon \frac{P_{k \mid k}^{-(i)}}{1+\left\|P_{k \mid k}^{-(i)}\right\|},
\end{aligned}
$$

where $\|\cdot\|$ is the frobenius norm of a matrix and $\epsilon>0$ is a relatively small constant. $\bar{H}_{k}^{(i)}, P_{k \mid k}^{-(i)}$, and $\bar{R}_{k}^{(i)}$ for the sensor $i$ can be calculated from the equations (16), (13), and (17) with local observation matrix $H_{k}^{(i)}$.

Similar to averaging consensus algorithm, adding consensus term in equation (34) will force local state estimators to reach a consensus. Subsequently, input estimator could be obtained from the innovation with the least squares estimation, which is unbiased for local sensor node $i$.

Algorithm 1 shows a message passing version of the distributed information consensus filter for simultaneous input and state estimation during one time step for the node $i$. The inverse of the covariance matrices and the state information estimates instead of measurements are transmitted for consensus fusion.

Remark 4: In consensus filter for simultaneous input and state estimation, we choose $K_{k}^{(i)}$ as the standard Kalman filter, while the proposed algorithm is obviously not the optimal filter for distributed estimation. In fact, the coupled estimates among the sensors makes it difficult to find an optimal solution minimizing the estimation error. The performance of this suboptimal distributed algorithm, which approximating the centralized optimal Gillijins-De Moor's algorithm will be illustrated by a example in the next section.

Remark 5: The choose of consensus gain $C_{k}^{(i)}$ is free, while a poor choice leads to either lack of consensus on estimation, or lack of stability of the error dynamics of the filter.

\section{Example}

Let the linear system under consideration be represented by a fourth-order model with order $n=4, m=1$, and matrices

$$
\begin{gathered}
A=\left[\begin{array}{rrrr}
-0.1 & 0.13 & 0.34 & 0 \\
-0.1 & -0.3 & -0.1 & 0.1 \\
-0.1 & 0 & 0 & 0.2 \\
0.21 & -0.1 & -0.3 & -0.5
\end{array}\right], \\
B=\left[\begin{array}{llll}
1 & 0 & 0 & 0
\end{array}\right]^{T} .
\end{gathered}
$$

where input $d_{k}$ and process noise $w_{k}$ are taken as the Gaussian white noises with zero mean and unit variance, respectively. We assume no information about $d_{k}$ is available for use. 


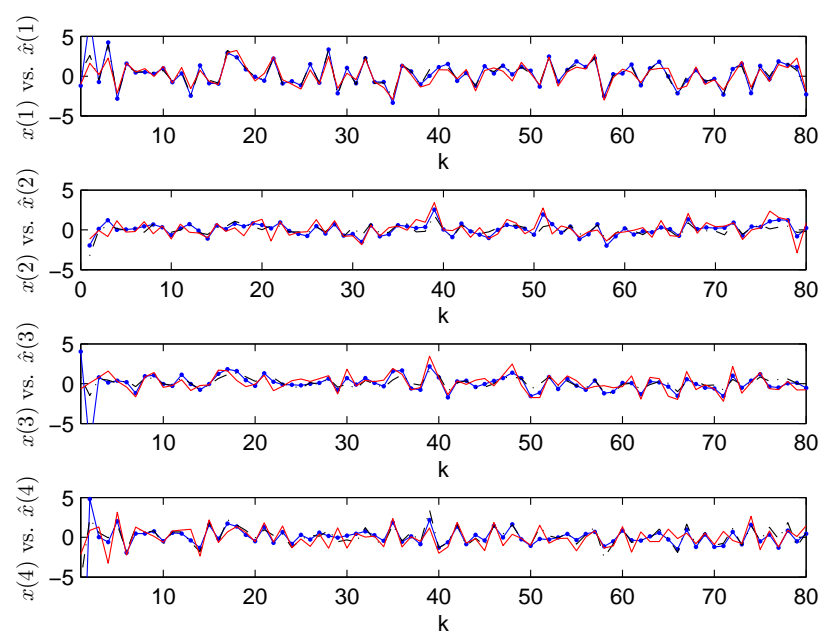

Fig. 1 Actual state values (red solid line), centralized state estimation (black dashed line), and distributed state estimation (blue dot-solid line). From top to bottom: the components of the state variable

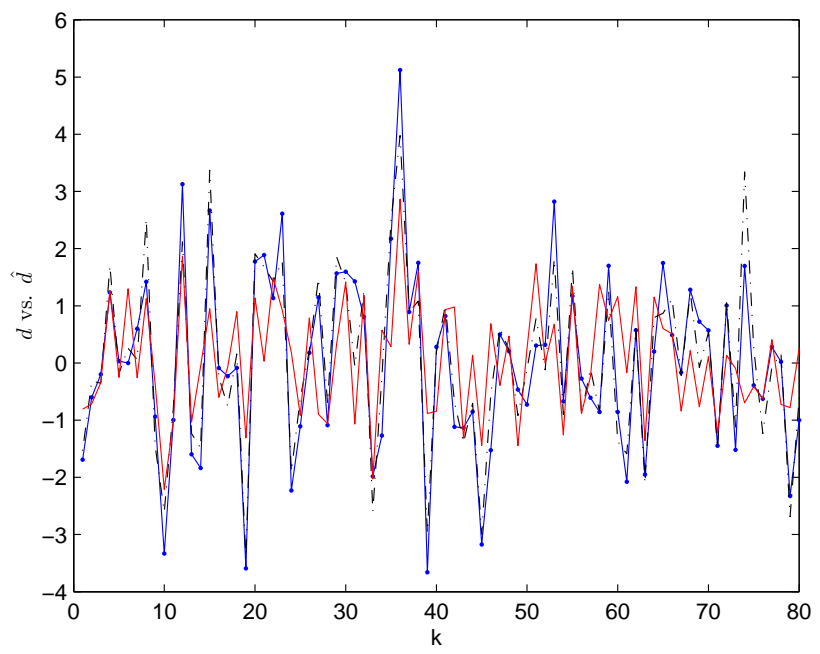

Fig. 2 Actual input values (red solid line), centralized input estimation (black dashed line), and distributed input estimation (blue dot-solid line) 
Table 1 Comparison between the 2-norm of input and state estimation errors by Algorithm 1 and Gillijns-De Moor algorithm

\begin{tabular}{ccc}
\hline & Algorithm 1 & Gillijns-De Moor algorithm \\
\hline $\begin{array}{c}\text { Input estimation } \\
\|\tilde{d}\|_{2}\end{array}$ & 4.3427 & 4.0323 \\
\hline State estimation & & \\
$\|\eta(1)\|_{2}$ & 0.9184 & 0.8932 \\
$\|\eta(2)\|_{2}$ & 3.1212 & 3.0452 \\
$\|\eta(3)\|_{2}$ & 1.0000 & 0.8550 \\
$\|\eta(4)\|_{2}$ & 5.1687 & 5.0015 \\
\hline
\end{tabular}

Two sensor nodes are located separately with the following partial observation matrices:

$$
\begin{aligned}
& H^{(1)}=\left[\begin{array}{llll}
1 & 1 & 0 & 0 \\
0 & 1 & 0 & 1
\end{array}\right], \\
& H^{(2)}=\left[\begin{array}{llll}
1 & 0 & 1 & 0 \\
0 & 0 & 1 & 1
\end{array}\right],
\end{aligned}
$$

with the measurement noise $v_{k}^{(i)}=0.02 I_{2}, i=1,2$.

In this sensor networks, the first sensor accesses to local measurement $z_{k}^{(1)}$ with noisy state components $x(1), x(2)$, and $x(4)$, while the second sensor accesses to local measurement $z_{k}^{(2)}$ with noisy state components $x(1), x(3)$, and $x(4)$. Clearly the system states are not fully observable with each single sensor nodes separately, but are fully observable by the centralized observation matrix $H=\left[H^{(1)} H^{(2)}\right]^{T}$.

In this experiment, we assume that two sensor nodes are interactive connected. The consensus gains are $C^{(i)}=0.5, i=1,2$, and the initial values for each sensor group are

$$
P_{0 \mid 0}^{+(i)}=10^{6} I_{4}, \bar{x}_{0 \mid 0}^{(i)}=\left[\begin{array}{llll}
2 & 2 & 2 & 2
\end{array}\right]^{T} .
$$

We compare the input estimation errors $\tilde{d}_{k}=d_{k}-\hat{d}_{k}$ and the state estimation errors $\eta_{k}$ between the distributed information filtering and the centralized optimal Gillijins-De Moor's algorithm. The corresponding 2-norm values of the estimation errors in one implementation are shown in Table 1. From Figure 1, Figure 2 and Table 1, we conclude that the performance of the proposed distributed consensus estimator is close to the optimal one.

The evolution of the disagreement term defined as

$$
\left\|\delta_{k}\right\|^{2}=\sum_{i=1}^{N}\left(\delta_{k}^{(i)}\right)^{2},
$$




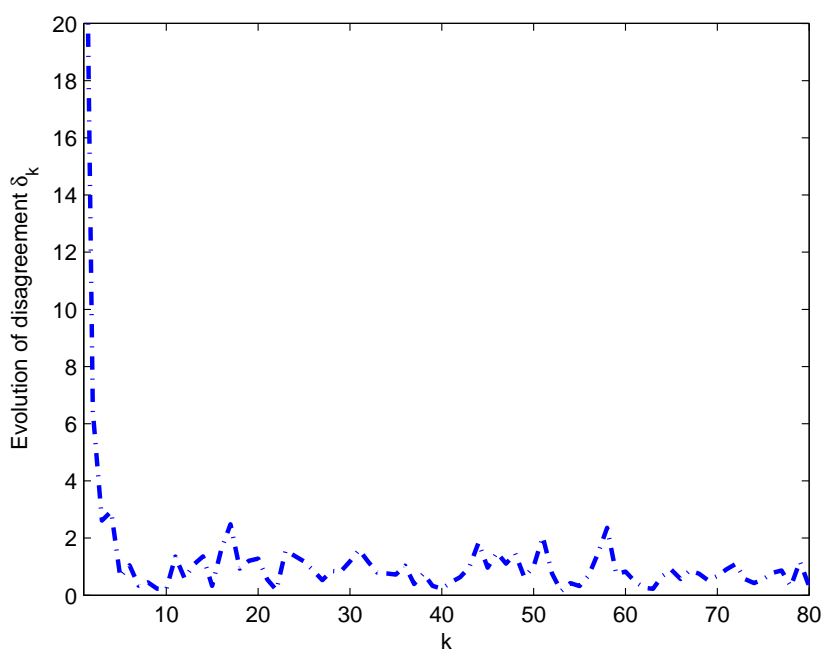

Fig. 3 Evolution of consensus disagreement $\delta_{k}$

where $\delta_{k}^{(i)}=\hat{x}_{k}^{(i)}-\frac{1}{N} \sum_{j} \hat{x}_{k}^{(j)}$, is depicted in Figure 3 which shows a relatively high disagreement at the beginning is bounded asymptotically.

\section{Conclusion}

In this paper, distributed filtering for simultaneous input and state estimation is presented with a consensus protocol. The local filtering algorithm is developed by integrating input estimation error into weighted local measurement and local information matrix. A consensus algorithm is developed using the averaging protocol which penalizes the disagreement in a dynamic manner. Simulation example is shown that the proposed distributed consensus filtering algorithm performs closely to the optimal centralized Gillijins-De Moor's algorithm.

Future work will consider applications of the distributed consensus filtering estimation to some autural systems, such as the networks of traffic flows with changing traffic demands, and hydrological and atmospheric transport models with unknown boundary conditions.

Acknowledgements This work was supported in part by Projects of International Cooperation and Exchanges of Natural Science Foundation of China (NSFC) under grant 61111130119, NSFC 60904069, the Doctoral Fund of Ministry of Education of China under grant 20091103120008, and the project of Communication University of China under grant YNG0710. X. Mao would like to thank the Royal Society of Edinburgh, the London Mathematical Society, the Edinburgh Mathematical Society. 


\section{References}

1. M.D.S. Aliyu, E.K. Boukas, Kalman filtering for affine nonlinear descriptor systems, Circuits Syst. Signal Process. 30, 125-142 (2011)

2. B.D.O. Anderson, J.B.Moore, Optimal Filtering, Englewood Cliffs, (NJ: Prentice-Hall, 1979)

3. M. Darouach, M.Zasadzinski, M.Boutayeb, Extension of minimum variance estimation for systems with unknown inputs, Automatica 39, 867-876 (2003)

4. M. Demetriou, Design of consensus and adaptive consensus filters for distributed parameter systems, Automatica 46, 300-311 (2010)

5. H. Fang, J. Wu, Y. Shi, Genetic adaptive state estimation with missing input/output data, Proceedings of the Institution of Mechanical Engineers, Part I, Journal of Systems and Control Engineering 224, 611-617 (2010)

6. S. Gillijns, B.De Moor, Unbiased minimum-variance input and state estimation for linear discrete-time systems, Automatica 43, 111-116 (2007)

7. S. Gillijns, B.De Moor, Unbiased minimum-variance input and state estimation for linear discrete-time systems with direct feedthrough, Automatica 43, 934-937 (2007)

8. C. Hsieh, Robust two-stage kalman filters for systems with unknown inputs, IEEE Trans. Autom. Control 45, 2374-2378 (2000)

9. U.A. Khan, J. Moura, Distributing the kalman filter for large-scale systems, IEEE Trans. Signal Processing 56, 4919-4935 (2008)

10. P. Kitanidis, Unbiased minimum-variance linear state estimation, Automatica 23, 775778 (1987)

11. X. Li, V. Jilkov, A survey of maneuvering target trackingCpart IV: decision-based methods, Proceedings of the SPIE Conference on Signal and Data Processing of Small Targets,(2002), pp.4728-4760

12. W. Liu, H. Tang, W. Fang, P. Ye, Estimation of the non-measurable state variables of a transcutaneous energy transmission system for artificial human implants using extended kalman filters, Circuits Syst. Signal Process. 28, 581-593 (2009)

13. R. Patton, P. Frank, R. Clark, Fault Diagnosis in Dynamic Systems: Theory and Application, (Prentice: Upper Saddle River, 1989)

14. S. Pillosu, A. Pisano, E.Usai, Consensus based decentralized estimation for linear plants with unknown inputs, Procedding of 49th IEEE Conference on Decision and Control, (2010), pp.6857-6862

15. R.O. Preda, D.N. Vizireanu, A robust wavelet based video watermarking scheme for copyright protection using the human visual system, Journal of Electronic Imaging 20, 13-22 (2011)

16. R.O. Preda, D.N. Vizireanu, Quantization based video watermarking in the wavelet domain with spatial and temporal redundancy, International Journal of Electronics 98, 393-405 (2011)

17. R.O. Preda, D.N. Vizireanu, A robust digital watermarking scheme for video copyright protection in the wavelet domain, Measurement 43, 1720-1726 (2010)

18. R.O. Preda, D.N. Vizireanu, Blind watermarking capacity analysis of MPEG2 coded video, Procedding of 8th International Conference on Telecommunications in Modern Satellite, Cable and Broadcasting Services, (2007), pp.465-468

19. R.O. Saber, Distributed kalman filter with embedded consensus filters, Proceeding of 44th IEEE Conference on Decision and Control, and European Control Conference, (2005), pp.8179-8184

20. R.O. Saber, J. Shamma, Consensus filters for sensor networks and distributed sensor fusion, Proceeding of 44th IEEE Conference on Decision and Control, and European Control Conference, (2005), pp.6698-6703

21. R.O. Saber, Kalman-consensus filter: optimality, stability, and performance, Proceeding of 48th IEEE Conference on Decision and Control, and Chinaese Control Conference, (2009), pp.7036-7042

22. M. Sharan, S.Raman, Weather and Climate: The M.P.Singh Vol. Part II, (Birkhauser: Basel, 2005)

23. Y. Shi, H. Fang, Kalman filter based identification for systems with randomly missing measurements in a network environment, International Journal of Control 83, 538-551 (2010) 
24. D. Simon, Optimal State Estimation: Kalman, $H_{\infty}$, and Nonlinear Approaches, (NJ: Jone Wiley and Sons, 2006)

25. S.S. Stankovic, M.S.Stankovic, D.M.Stipanovic, Consensus based overlapping decentralized estimator, IEEE Trans. Autom. Control 54, 410-415 (2009)

26. S.S. Stankovic, M.S.Stankovic, D.M.Stipanovic, Consensus based overlapping decentralized estimation with missing observations and communication Faults, Automatica 45 , 1397-1406 (2009)

27. L. Xiao, S.Boyd, Fast linear iterations for distributed averaging. Systems and Control Letters 53, 65-78 (2004)

28. L. Zhang, R.Sengupta, Distributed kalman consensus filter for distributed parameter systems with unknown boundary conditions, submit to the 51th IEEE Conference on Decision and Control, (2012) 